\title{
SELECCIÓN DE HERRAMIENTAS DISCURSIVAS PARA EL ANÁLISIS DEL LENGUAJE JURÍDICO
}

\author{
Marcela AguirREZABALA \\ Profesora Adjunta de Historia Constitucional \\ Departamento de Humanidades \\ Universidad Nacional del Sur (UNS). Argentina \\ maraguirrezabala@bvconline.com.ar \\ Natalia Paula FANDUZZI \\ Asistente de Docencia de Historia Constitucional \\ Departamento de Humanidades \\ Universidad Nacional del Sur (UNS). Argentina \\ nfanduzzi@gmail.com
}

\begin{abstract}
RESUMEN
En este artículo nos proponemos introducir al lector en el análisis del lenguaje jurídico desde la perspectiva sistémico-funcional de los estudios del discurso. Nuestro propósito es retomar los aportes de distintos especialistas con el objetivo de configurar una caja de herramientas para el análisis del lenguaje legal, entendido como lenguaje de especialidad, que resulte útil a quienes deseen introducirse en su estudio. Para ello se estudian los conceptos de género y registro, se caracterizan las particularidades formales y se analiza la variedad interna del lenguaje observado. Estos aportes posibilitan hacer un mapeo del texto jurídico a través de un enfoque situacional focalizado en la exploración de su configuración contextual, pero sin olvidar su estructura cognitiva y su pretensión comunicativa.
\end{abstract}

Palabras clave: Lenguaje jurídico, estudios del discurso, perspectiva sistémicofuncional.

\section{ABSTRACT}

In this article we intend to introduce the reader to the study of legal language from the functional-systemic perspective of the studies of speech. Our aim is to retake the contributions of different specialists so as to create working tools for the study of legal language, understood as specialist language, so that it is useful for those who wish to devote to its study. To that purpose we will see the concepts of genre and register once again, we will feature the formal peculiarities and we will analyse the internal variety of the language focus of study. Such contributions make it possible to map out the legal text through a situational approach focused on the exploration of its contextual configuration without forgetting its cognitive structure and its communicative intention.

Keywords: Legal language, speech studies, functional-systemic perspective. 


\section{ZUSAMMENFASSUNG}

In diesem Artikel wollen wir den Leser in die juristische Sprachanalyse aus systematisch-funktionaler Perspektive mittels Diskursuntersuchungen einführen. Unter Berücksichtigung der Beiträge verschiedener Fachwissenschaftler verfolgen wir das Ziel, Handwerkszeug zusammenzustellen, um die juristische Sprache als Fachsprache analysieren zu können und denjenigen eine Handreichung zu bieten, die eine Einführung in die juristische Sprachanalyse suchen. Hierzu werden die Begriffe der jeweiligen Rechtsgattung und der Protokollaktenführung durchgesehen; es werden die formalen Besonderbeiten herausgestellt und es wird die interne Vielfalt der Sprache analysiert. Diese Beiträge ermöglichen die Erstellung einer juristischen Landkarte juristischer Texte durch eine situationsbezogene Betrachtung, die eine Untersuchung der kontextuellen Beschaffenheit fokusiert, jedoch obne die jeweilige kognitive Struktur und die kommunikative Absicht aus dem Auge zu verlieren.

Schlüsselwörter: Juristische Sprache, Diskursanalysen, Systematisch-funktionale Perspektive.

SUMARIO: I. INTRODUCCIÓN.-II. ANÁLISIS DE GÉNERO Y REGISTRO DEL DISCURSO.-III. PARTICULARIDADES DEL LENGUAJE JURÍDICO.-IV. VARIEDAD INTERNA DEL LENGUAJE LEGAL.V. ACERCA DE ALGUNOS GÉNEROS ESPECÍFICOS DEL LENGUAJE JURÍDICO.-1. Las disposiciones legales.-2. Los casos legales.-VI. EL TEXTO JUDICIAL COMO TEXTO DE PODER.-VII. CONSIDERACIONES FINALES.-VIII. BIBLIOGRAFÍA.

\section{INTRODUCCIÓN}

El lenguaje jurídico, en tanto lenguaje de especialidad, presenta rasgos distintivos que permiten analizarlo aisladamente. Su utilización, no obstante, excede ampliamente su ámbito profesional de origen y en la actualidad son múltiples las disciplinas que centran su interés en temas vinculados con la impartición de justicia. Los procesos judiciales y de promulgación de leyes y disposiciones, así como los casos particulares, entre otros, son estudiados por científicos sociales en búsqueda de un conocimiento más acabado de los conflictos aludidos, los agentes involucrados y los contextos históricos en los que éstos interactúan.

De lo expuesto se desprende la necesidad de contar con ciertas herramientas discursivas para el análisis del lenguaje jurídico expresado en los textos de la especialidad. En este artículo nos proponemos introducir el análisis de este lenguaje particular desde la perspectiva sistémico-funcional de los estudios del discurso. El carácter tentativo de nuestra propuesta se expresa en su formulación descriptiva que retoma los aportes de dis- 
tintos especialistas con la intención de configurar una caja de herramientas ${ }^{1}$ para el análisis del lenguaje legal que resulte útil a quienes deseen introducirse en su estudio.

De este modo, iniciamos nuestro planteamiento con una aproximación a los conceptos de género y registro, señalando las nociones que consideramos operativas para lograr nuestro objetivo. Seguidamente, nos sumergiremos en la caracterización de las particularidades del lenguaje jurídico entendido como un lenguaje de especialidad con manifestaciones formales propias. En tercer lugar, analizaremos la variedad interna del lenguaje legal a partir de la diferenciación de dos de sus géneros específicos: las disposiciones y los casos legales, aislando en cada caso su configuración contextual, su propósito comunicativo y su estructura cognitiva.

Finalmente incluimos un apartado donde planteamos sintéticamente algunas de las implicaciones derivadas de considerar al texto jurídico como texto de poder. Esta última sección únicamente se propone manifestar algunas de las cuestiones que se prefiguran a lo largo del trabajo como expresión de una problemática a desarrollar en el futuro.

\section{ANÁLISIS DE GÉNERO Y REGISTRO DEL DISCURSO}

El análisis discursivo de registro y género supone, según Eggins y Martin $^{2}$, la concreción de dos pasos secuenciales. En primer lugar, la descripción de las pautas lingüísticas del texto - específicamente las pautas léxicas, gramaticales y semánticas, tales como la formalidad textual, las expresiones de actitud y el conocimiento supuesto en los mismos-. En segundo término, la explicación de las diferencias lingüísticas identificadas anteriormente atendiendo al contexto situacional en que se producen y

${ }^{1}$ Utilizamos el concepto «caja de herramientas» en el sentido de algunos teóricos franceses como Deleuze que se refirió a la teoría como «caja de herramientas»: «Eso es una teoría, exactamente como una caja de herramientas. No tiene nada que ver con el significante», G. Deleuze y M. Foucault, Un diálogo sobre el poder. Michel Foucault utilizó el concepto «caja de herramientas» teóricas para señalar un trabajo intelectual no concluido, sino como «pistas de investigaciones, ideas, esquemas, punteados, instrumentos», separándose de las metodologías canónicas absolutas. Escribe Foucault: «Todos mis libros [...] son, si se quiere, pequeñas cajas de herramientas», M. Foucault, Saber y Verdad (1991). En un sentido similar, Gerard Genette utilizó el concepto «bricolaje» elaborado por Levi-Strauss para aplicarlo a la crítica literaria.

2 S. EgGins y J. Martin, «Géneros y registros del discurso», en T. VAn DijK (coord.), El discurso como estructura y proceso. Estudios del discurso I. Una introducción multidisciplinaria, vol. 1, Barcelona, 2003, pp. 335-372. 
circulan los textos. Los autores intentan, siguiendo estos pasos, fundamentar la variación lingüística vinculando el discurso con las variables específicas del contexto social y cultural en el cual se materializa dicho discurso. Registro y género son entonces las nociones que permiten explicar el significado y la función de la variación entre los textos.

Las dimensiones contextuales desarrolladas desde esta perspectiva teórica vinculadas con elecciones de vocabulario y estructura son básicamente tres: 1) modo, alude a la relación del autor del texto con su audiencia; 2) tenor, refiere el rol del autor en el texto y a sus expresiones de actitud, y 3) campo, vinculado con el conocimiento supuesto en el texto, las expresiones técnicas y las referencias inter textuales. Más adelante retomaremos esta clasificación profundizándola y brindando ejemplos específicos de la ocurrencia de estas dimensiones en el lenguaje jurídico.

A su vez, la articulación vigente entre las pautas gramaticales y las dimensiones contextuales permite definir a los textos como semánticamente multidimensionales, caracterizándolos, según Eggins y Martin³ , como

«un entramado compuesto de muchas hebras diferentes portadoras de significado que actúan de manera simultánea [...] Por un lado, el texto aporta significados sobre una realidad [significados ideativos] [...] También habla de las actitudes del autor con respecto a su tema y de su relación de rol con los lectores. Ésos son los significados interpersonales del texto. Finalmente, a través de la hebra de los significados textuales, el texto dice algo acerca de cómo está organizado como acontecimiento lingüístico».

En relación con la multiplicidad de significados contenida en los textos jurídicos resulta significativo señalar los mecanismos que determinan la coherencia de los mismos, a fin de comprenderlos como una unidad semántica. Mecanismos tales como los recursos cohesivos del lenguaje y los que devienen de la estructura genérica realizada en el texto.

Por su parte, se entiende por registro a las selecciones léxicas, gramaticales y fraseológicas. El registro es la variedad funcional del lenguaje; la sustancia que adquiere forma en el texto. Es el correlato de una versión semántica como potencial de significado vinculado con ciertas situaciones contextuales específicas que se realizan en ciertos lenguajes. El concepto de registro asocia entonces las nociones de lenguaje y de contexto. Según Eggins y Martin ${ }^{4}$ :

${ }^{3}$ Ibid., pp. 339-340.

${ }^{4}$ Ibid., p. 340. 
«El concepto de registro es una explicación teórica de la elaboración de sentido común que indica que usamos el lenguaje de modos diferentes en situaciones diferentes. Para expresarlo más técnicamente, se puede ver que las dimensiones contextuales producen un impacto en el lenguaje que hace que ciertos significados, así como sus expresiones lingüísticas, sean más probables que otros».

Por su parte, se identifica a los géneros del discurso como «tipos relativamente estables de emisiones interactivas» ${ }^{5}$ relacionadas con un propósito social determinado. Los distintos géneros exhiben varios patrones de similitud; el primero en importancia es el propósito, luego la estructura, el estilo, el contenido y la audiencia pretendida.

El género comprende así la correspondencia existente entre estructura, contenido y registro, donde el contenido es expresado en término de funciones/propósitos comunicativos. Los diferentes géneros son el correlato de los distintos modos de utilizar el lenguaje según las tareas, culturalmente definidas, que se deseen cumplir. La relación entre contexto y género, no obstante, es definida como probabilística, más que como determinista, por lo que las distintas etapas o pasos desplegados en la consecución de las tareas propuestas también lo serán.

El estatus de las elecciones o pasos obligatorios es fundamental, ya que estos últimos son los que definen el género. Los elementos opcionales tienen una aplicabilidad más amplia. La aparición de todos ellos coincide con nuestra percepción de un texto completo y se asocia con la relación de previsibilidad enunciada anteriormente.

Por otra parte, el criterio para definir a los elementos estructurales es semántico. El texto, así comprendido, es lenguaje realizando un determinado trabajo en un contexto específico. El texto es expresión de la acción social que se desarrolla en ese contexto, como por ejemplo: prohibir, permitir, etc. En este sentido, el lenguaje se organiza funcionalmente en relación con las categorías contextuales, por lo que cada meta función (organización del lenguaje del texto) puede asociarse con un registro específico (organización del contexto) ${ }^{6}$. De este modo el significado interpersonal, los recursos de interacción, se vincula con el tenor o estructura de roles; el significado ideativo o los recursos para construir contenido, con el campo o acción social, y los recursos para organizar los textos o significado textual, con el modo u organización simbólica.

5 Ibid., p. 342.

${ }^{6}$ Ibid., p. 347.

Foro, Nueva época, vol. 15, núm. 2 (2012): 105-123 
Otros conceptos importantes para la perspectiva sistémico-funcional son los de configuración contextual y potencial de estructura genérica. La configuración contextual se vincula con la estructura de la situación comunicativa, es el conjunto particular de valores específicos asignados a las variables de campo, tenor y modo mencionadas más arriba. La configuración contextual da cuenta así de los atributos del contexto de una acción social determinada. Siguiendo lo expuesto por $\mathrm{Halliday}^{7}$, dichas variables pueden entenderse como:

«1. Campo, la acción social: lo que está pasando, la naturaleza de la acción social que está ocurriendo, es decir, en qué están ocupados los participantes, donde el lenguaje es como un componente esencial. 2. Tenor, la estructura de roles: quién está participando, la naturaleza de los participantes, sus posiciones y roles, es decir, qué tipos de relaciones de rol existen entre los participantes, incluyendo relaciones permanentes y temporales de uno u otro tipo, tanto los tipos de roles discursivos que adoptan en el diálogo como todo el grupo de relaciones socialmente significativas en las que están involucrados. 3. Modo, la organización simbólica: qué papel desempeña el lenguaje, qué es lo que esperan los participantes que el lenguaje haga por ellos en la situación, es decir, la organización simbólica del texto, la posición que éste ocupa y su función en el contexto, incluyendo el canal de expresión (si es hablado, escrito o una combinación de ambos) y también el modo retórico, lo que se logra por medio del texto en términos de categorías como persuasivo, expositivo, didáctico y otros por el estilo».

Las implicaciones de la utilización de estas herramientas para el estudio del lenguaje legal son múltiples según el caso en estudio. Nos interesa específicamente destacar, en esta época de abordajes interdisciplinarios, que su utilización por parte de distintos especialistas permite tanto el establecimiento de pautas de trabajo comunes, como la puesta a prueba constante y desde diferentes perspectivas de dichos instrumentos analíticos. Ejercicio este último que posibilitaría, a nuestro entender, el enriquecimiento de los resultados obtenidos en las investigaciones llevadas a cabo por los científicos sociales.

${ }^{7}$ Ibid., p. 346. 


\section{PARTICULARIDADES DEL LENGUAJE JURÍDICO}

A esta altura estamos en condiciones de afirmar que el lenguaje legal constituye un lenguaje de especialidad porque remite a conjuntos de registros o variedades funcionales propias de un ambiente profesional particular. El lenguaje legal se diferencia del resto en la sintaxis determinada o controlada que emplea, en su alto nivel de abstracción, en sus formas de expresión —que manifiestan un uso singular del lenguaje natural-y en los vínculos que establece con sus interlocutores. Por otra parte, al tratarse de un lenguaje de especialidad, tal como podría ser el de las ciencias experimentales, no puede sustraerse de características compartidas como lo son el uso de una sintaxis formal, una terminología propia o técnica, y el recurso de utilización de símbolos artificiales. Como sostienen Duarte y Martínez ${ }^{8}$ :

«El lenguaje jurídico es sin duda un tecnolecto y comparte con los otros lenguajes de especialidad la precisión (univocidad terminológica y, por tanto, ausencia de sinonimia), la formalidad (neutralidad afectiva y carencia de elementos emotivos), la impersonalidad y el carácter eminentemente funcional del lenguaje (la comunicación tecnolectal es básicamente eficaz y no persigue ningún objetivo de tipo estético)».

En este sentido el lenguaje jurídico está constituido por enunciados de tipo performativo que indican conductas o normas, ya sea calificándolas y/o prohibiéndolas ${ }^{9}$, y a cada elemento formal característico puede asociársele una finalidad y/o aplicación determinada. Algunas de las manifestaciones del lenguaje jurídico señaladas por Duarte y Martínez ${ }^{10}$ son: 1) formalidad y gentileza (se busca conciliar la eficacia de las decisiones con el carácter oficial de los organismos judiciales y administrativos que utilizan este lenguaje); 2) objetividad y subjetividad (aun cuando existe un componente subjetivo inevitable, es la apariencia de objetividad lo que confiere credibilidad y hace convincente a la presentación); 3) dependencia de textos legales (la remisión a otros textos legales muestra el apego a la normativa vigente, la repetición se utiliza para otorgar fuerza al documento y la existencia de un diccionario jurídico propio caracteriza al universo jurí-

\footnotetext{
${ }^{8}$ C. Duarte y A. Martínez, El lenguaje jurídico, Buenos Aires, 1995, p. 30.

9 N. Fóscolo y M. Schilardi, Materialidad y poder del discurso. Decir y hacer jurídicos, Mendoza, 1996, p. 26.

${ }^{10}$ Ibid., p. 31.
} 
dico); 4) funcionalidad (este aspecto hace a la precisión de los términos empleados, sólo se tiene en cuenta la información necesaria, apelando a la evidencia de lo dicho, a la claridad y a la relevancia del caso), y 5) organización textual (apunta a la función comunicativa, hay una organización estructural que es habitual en textos de naturaleza jurídica, como por ejemplo: Hechos.—De lo que resulta, y considerando.—Resuelvo).

\section{VARIEDAD INTERNA DEL LENGUAJE LEGAL}

Vijay Bhatia en Legal discourse in professional settings ${ }^{11}$ presenta una definición alternativa de la noción de potencial de estructura genérica enunciada en el primer apartado aplicable al lenguaje legal, la cual adscribimos y reproducimos seguidamente. Según el autor, el término lenguaje legal abarca varios géneros útiles y distinguibles que dependen de los propósitos comunicativos que tienden a cumplir, de los ambientes o contextos en los cuales son usados, de los eventos o actividades comunicativas a los que están asociados, de la relación social o profesional entre los participantes que toman parte en esas actividades o eventos, del conocimiento anterior que los participantes traen a la situación en la cual ese evento particular está involucrado, y de un número de otros factores.

Bhatia identifica también varios géneros usados en una variedad de ambientes legales: en el medio escrito (casos, juicios, legislación, contratos, acuerdos, etc.) y en el medio oral (juntas abogado-cliente, indagación fiscal-testigo, etc.)

Dentro de los géneros legales reconoce asimismo dos variaciones principales:

1. Diferentes clases de sistemas legales: 1.1) Derecho consuetudinario (ley común: prefiere la particularidad; el propósito de su diseñador es no ser malentendido por la comunidad especialista); 1.2) Ley civil (prefiere la generalidad; el propósito de su diseñador es ser comprendido por lectores comunes) y 1.3) Ley Shariat (mundo musulmán).

2. Distintas manifestaciones formales del discurso legal dentro de un mismo sistema: 2.1) extensión de los términos (certeza/elegancia); 2.2) grado de ortodoxia en el uso de recursos lingüísticos; 2.3) dispositivos de mapeo textual o de planeamiento de textos (son estrategias de dis-

11 V. Bhatia (ed.), Analysing Genre: language use in profesional settings, 1998, pp. 101-144. 
curso que brindan coherencia textual relacionando un aspecto del texto con otro, ayudando así al diseñador a reducir la carga de información a un punto particular en la sintaxis de una declaración legislativa).

\section{ACERCA DE ALGUNOS GÉNEROS ESPECÍFICOS DEL LENGUAJE JURÍDICO}

Consideraremos ahora, siguiendo la propuesta de Bhatia, dos géneros del medio escrito para su análisis en profundidad: legislación y casos.

En principio, las disposiciones y los casos legales comparten: escenarios de uso o comunidad profesional, participantes en sus actividades comunicativas profesionales, uso como autoridad legal en la negociación de justicia, y la forma en que codifican y representan los procesos legales. Su aplicabilidad se vincula con la interacción vigente entre el mundo exterior y el mundo modelo — subyacente al discurso jurídico- que genera una situación contextual con características propias.

\section{GRÁFICO 1}

Aplicabilidad de casos y disposiciones legales

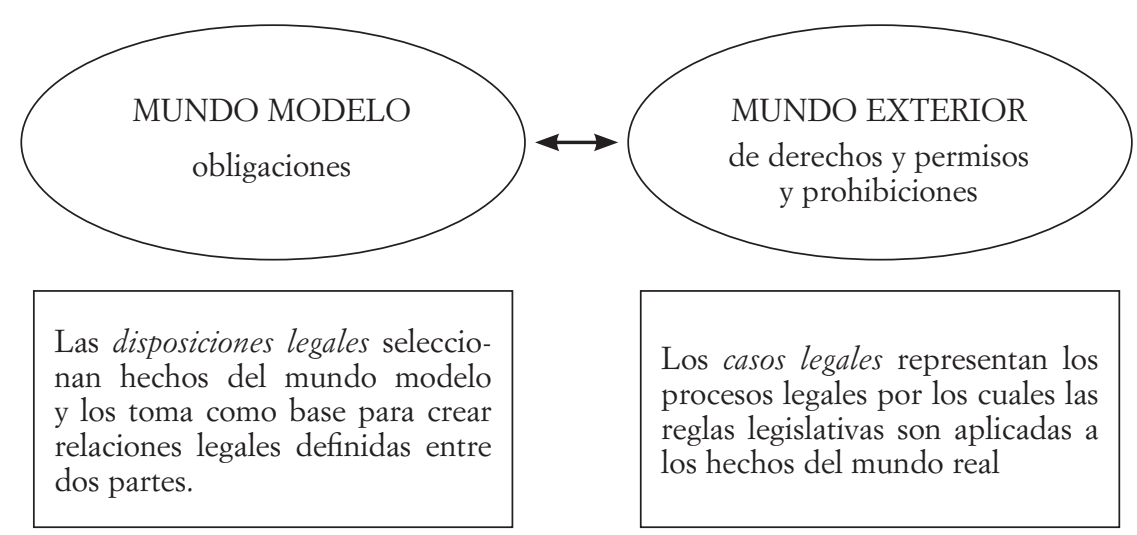

\section{Las disposiciones legales}

Inicialmente nos proponemos responder a la pregunta: ¿por qué son las disposiciones legales escritas de la manera que lo son? Para ello se tendrán en cuenta los propósitos comunicativos que estas disposiciones están desti- 
nadas a servir y las coacciones impuestas en el delineamiento de las mismas. De este modo, y siguiendo con la reseña de la propuesta de Bhatia, se explicitará el potencial de estructura genérica para los casos seleccionados.

El propósito comunicativo de las disposiciones legislativas se relaciona con su función general directiva. Esta escritura busca imponer obligaciones y conferir derechos. En las disposiciones legislativas, el diseñador parlamentario es únicamente el escritor del decreto legislativo, el cual se origina en las deliberaciones de un Parlamento en el cual él no está nunca presente. Asimismo, el documento es destinado a los ciudadanos comunes, aunque los lectores reales son abogados y jueces, quienes, a su vez, son los responsables de interpretar estas disposiciones para los ciudadanos comunes. Por estos motivos el diseñador intenta producir disposiciones no sólo claras, precisas y sin ambigüedad, sino también inclusivas de la totalidad.

Gunnarson ${ }^{12}$ distingue tres tipos de reglas legislativas:

a) Las reglas de acción. Son aplicables sólo a un conjunto de descripciones específicas de casos y están principalmente destinadas a imponer deberes y obligaciones, dar derechos, prohibir acciones, asignar poder a ciertos miembros de los cuerpos del ejecutivo u otras partes y a establecer la ley o sólo las penalidades impuestas sobre acciones específicas.

b) Las reglas de estipulación. Definen el dominio de aplicación de un decreto particular o cualquier sección de él.

c) Las reglas de definición. Están destinadas a proveer explicación terminológica.

La mayor parte de la legislación consiste en reglas de acción más que de los otros dos tipos. Nuestra discusión aborda primordialmente las denominadas disposiciones legislativas. Las propiedades sintácticas predominantes de las mismas son: longitud de oración, carácter nominal, frases preposicionales complejas (preposición + sustantivo + preposición) y expresiones binominales y multinominales (por ejemplo, a menos y hasta).

Paralelamente el autor identifica tres variables de análisis específicas de las disposiciones legales:

a) Descripciones de caso inicial. El tema legal es convencionalmente demorado por la introducción de una descripción de caso larga en la forma de una cláusula adverbial comenzando con «donde», «si» o, a veces, «cuando». Su importancia reside en que es absolutamente crucial para el escritor especificar la clase de descripción(es) de caso a la cual la regla se aplica.

${ }^{12}$ Ibid., pp. 104-105. 
b) Requisitos en disposiciones legislativas. La característica más relevante de la declaración legislativa es el uso de requisitos sin los cuales la disposición perderá su naturaleza esencial. Los requisitos adjuntos restringen a la normativa.

c) Discontinuidades sintácticas. No es simplemente la presencia de requisitos lo que hace de las disposiciones legislativas un género interesante, sino también la forma en que estos requisitos se insertan dentro de la sintaxis de una oración legislativa.

Por su parte, en la estructuración cognitiva de las disposiciones legislativas, en lo referido al proceso de hacer leyes, se delinean dos aspectos importantes:

1. La cláusula de disposición principal:

1.1. El sujeto legal (la persona o la parte que es el sujeto de la disposición).

1.2. La acción legal (la naturaleza del poder o derecho que le es dado hacer o prohibido de hacer, que surge de la disposición).

2. Los requisitos:

2.1. Preparatorios [dan forma a la descripción del caso(s) al cual el estatuto de ley se aplica].

2.2. Operacionales (dan información adicional acerca de la ejecución u operación del estatuto de ley).

2.3. Referenciales (especifican la naturaleza esencial inter-textual de la disposición legislativa).

Para una descripción estructural adecuada del género lo más apropiado es pensar en términos de una estructura cognitiva interactiva de estas dos partes: la cláusula de disposición principal y los requisitos, más que en una organización lineal de los actos como los encontrados en otros géneros. El análisis de estructuración cognitiva es interactivo, en el sentido de que el movimiento de requisitos interactúa con diversos aspectos del movimiento de la cláusula legislativa en varias posiciones respondiendo a un número de cuestiones que pueden ser legítimamente preguntadas en el contexto. La función principal de estos requisitos o condiciones es hacer a la disposición legislativa precisa, clara, no ambigua e inclusiva.

En este mismo sentido, y siguiendo el modelo de Perelman ${ }^{13}$, se pueden distinguir tres tipos de auditorio: «El primero está constituido por

13 R. MARAFIOTI, Los patrones de la argumentación. La argumentación en los clásicos y en el siglo XX, Buenos Aires, 2003, p. 101.

Foro, Nueva época, vol. 15, núm. 2 (2012): 105-123 
toda la humanidad o, al menos, por todos los adultos y normales, es el auditorio universal; el segundo es el formado, desde el punto de vista del diálogo, por el único interlocutor al que el argumentador se dirige, y el tercero es el integrado por el propio sujeto cuando delibera o evoca interiormente acerca de las razones de sus actos».

Para finalizar, cabe reflexionar sobre los roles asumidos por los legisladores en el discurso legal. El legislador aparece como un sujeto colectivo cuyo decir sobre el objeto representado por la cultura jurídica señala a través de su discurso jurídico —entendido como un enunciado global— lo conveniente para el mantenimiento del sistema por él mismo definido. El legislador es, pues, un «actuante-instaurador de las formas jurídicas, pero también actuante-controlador cuando aparece la justicia» ${ }^{14}$.

\section{Ejemplo de disposición legislativa}

Corresponde a la 32. ${ }^{\circ}$ reunión, 3. ${ }^{a}$ Sesión Ordinaria (continuación), 17 de agosto de 1994, en Diario de Sesiones de la Convención Nacional Constituyente, Santa Fe-Paraná, Secretaría Parlamentaria, tomo IV, 1994, pp. 4377-4380.

«Sr. Presidente (Pierri).--Tiene la palabra la señora convencional por el Chubut.

Sra. MARCOLONI.-Señor presidente: brevemente intentaré hacer conocer la opinión del bloque de la Unión Cívica Radical respecto del tema habilitado por el artículo $3 .^{\circ}$, punto J, de la Ley 24.309, que estamos analizando en este momento.

Nuestra Constitución establece como forma de gobierno la republicana y representativa, por lo que se hace evidente que el fundamento para establecer el derecho electoral surge de las entrañas mismas de esta Constitución que estamos modificando y reposa sobre el principio por el cual la autoridad nace del pueblo y se ejerce en representación de la soberanía popular para el bienestar de toda la comunidad.

El sufragio, en tanto y en cuanto permite elegir a nuestros gobernantes, es una expresión acabada y perfecta del ejercicio de la libertad individual.

Reconocemos al 22 de mayo de 1810 por el voto indirecto que el Cabildo expresó en nombre del pueblo, a través del cual destituye al virrey y nombra a la primera junta de gobierno que integraron, entre otros, Saavedra y Moreno. También con una forma indirecta de elección se llegó a constituir la Asamblea de 1813 y a designar los diputados de 1815.

${ }^{14}$ N. Fóscolo y M. Schilardi, op. cit., p. 37. 
Recién en 1857, en cumplimiento de lo establecido por la Constitución de 1853, se dicta la primera ley regulatoria de elecciones nacionales que lleva el número 140 y por la cual se determinó un sistema de voto de carácter voluntario, público y reducido a los varones mayores de dieciocho años, en la que se incluía también un sistema de lista completa.

Finalmente, el 12 de octubre de 1910 Roque Saénz Peña advierte: "No es suficiente garantizar el sufragio, tenemos el deber de crear y movilizar al votante". Con este concepto patentizado en la Ley 8.871, el 10 de febrero de 1912, se modifica sustancialmente la situación electoral al establecerse el sufragio secreto, universal y obligatorio para todos los ciudadanos varones. Como dijera el señor convencional Cardesa en la exposición anterior, en 1951 se introdujo el sistema de circunscripciones uninominales. En 1957 el sistema que se incorpora es el de representación proporcional que nosotros llamamos D'Hont.

También decimos en este dictamen que un hombre equivale a un voto, por lo cual se tiene en cuenta el padrón electoral en el que figuran los habilitados con sus domicilios y número de documento. Todas las personas que componen el cuerpo electoral se encuentran en las mismas condiciones. Éste es un principio pilar que, junto con la universalidad, caracterizan el sistema democrático. Toda persona, por el sólo hecho de serlo, tiene derecho a votar.

El voto femenino fue establecido en la Argentina por la Ley 13.010, sancionada en 1947, aunque recién se puso en práctica con la Ley 14.302, de 1951, como expresara anteriormente. En 1983 el cuerpo electoral estuvo integrado por un 53 por 100 de mujeres. Sin embargo, los más altos organismos colegiados muestran aún una dispar presencia de mujeres en situación de gravitar sobre el desarrollo de políticas fundamentales para la vida de nuestro país. Es por ello que propiciamos que se establezcan acciones positivas tanto en cuanto a la regulación de los partidos políticos como en la sanción del régimen electoral, como forma de garantizar una integración más plena e igualitarias y con posibilidades ciertas de acceso a cargos de responsabilidad pública. Para finalizar voy a decir con Badeni: "Es a través del reconocimiento y ejercicio de los derechos políticos que opera la participación verdadera de los individuos en el proceso del poder"». (Aplausos.)

\section{Los casos legales}

Se entiende por casos legales a las versiones resumidas de los juicios destinadas a fomentar la adquisición de las habilidades de razonamiento legal, habilidades argumentativas y la formación de decisiones por parte de los agentes que componen el campo jurídico. 
El discurso jurídico es referencial: «En este caso se trata de una elaboración ideológica, entendida como cobertura discursiva del mundo, el cual se da como el mundo social mismo, como la "realidad" y como si él fuera anterior a la palabra (el discurso legislativo) que lo articula» ${ }^{15}$.

\section{Ejemplo de caso legal}

[«La vendedora ha incurrido en un incumplimiento contractual ofreciendo en venta un inmueble con calefacción y agua caliente central y un jardín de 90 metros de uso exclusivo, características que el inmueble no tenía cuando los Sres. De Elizalde tomaron posesión.»] [Hechos]

[«En consecuencia, coincido con la sentenciante en que deben ser resarcidos en la medida en que ha mermado el valor de la propiedad.

Se queja también la apelante del monto fijado en la sentencia de \$32.000.

El Sr. Juan A. Nicodemo, ingeniero civil, comprobó en dos visitas al departamento que el jardín del contrafrente, de una superficie de 100,71 metros cuadrados, no es de uso exclusivo de la familia De Elizalde y calcula la diferencia de valor en $\$ 10.000$.

Con respecto a la diferencia de valor entre el departamento con y sin servicio de calefacción central, se remite al informe del instalador Oscar C. Guijo, quien cotiza los trabajos e instalación completa en $\$ 32.000$.

La apelante critica el informe pericial, aduciendo que carece de sustento técnico.

En este sentido ha dicho la sala que "si bien las normas procesales no otorgan al dictamen pericial el carácter de prueba legal cuando éste comporta la necesidad de una apreciación específica del saber del perito, para dejarlo de lado es de rigor, en principio, valorar elementos que permitan advertir fehacientemente el error o el insuficiente aprovechamiento de los conocimientos científicos que debe tener el perito por su profesión o título habilitante".»] [Argumentación]

[«Por otro lado, cuando la peritación aparece fundada en principios técnicos inobjetables, la sana crítica aconseja, frente a la imposibilidad de oponer argumentos científicos de mayor peso, aceptar las conclusiones de aquélla (conf. C. Nac. Civ., sala C, 12/8/1983, L 288962; íd., 29/2/1984, L 2382; íd., 14/11/1988, L 36226; íd., 23/8/1994, L 130191, votos del Dr. Alterini; íd., 17/4/2001, Pilipezuk, Hugo F. v. Vega, José M. s/daños y perjuicios, L 312438)» ${ }^{15}$.] [Sentencia]

15 Ibid., p. 27.

${ }^{16}$ LeXIs NexIs, Jurisprudencia Argentina, Compraventa, 15 de enero de 2003, JA 2003-1, fasc. 3, pp. 55-56. 
El ejemplo seleccionado corresponde a una sentencia emitida en segunda instancia que reconsidera el caso en cuestión revalidando lo establecido inicialmente por la Cámara Nacional Civil, Sala C. Consideramos que el texto escogido representa una muestra adecuada, a pesar de su carácter fragmentario, por dos motivos: a) su análisis externo evidencia el contexto profesional de uso de los casos legales que determina su propósito comunicativo, y b) su análisis interno - por tratarse de la reconsideración de lo actuado anteriormente- explicita las operaciones habituales en la comunidad profesional para la negociación de justicia (véase en especial el desarrollo de la argumentación).

En el ejemplo seleccionado se observan, asimismo, los distintos actos o movimientos que caracterizan la estructura cognitiva genérica de los casos legales y posibilitan su interpretación estructural. A saber, identificación del caso, establecimiento de los hechos, argumentación y sentencia (señalados entre corchetes).

El propósito comunicativo de los casos legales también se ajusta al potencial de estructura genérica definido por Bhatia, ya que la publicación de este tipo de textos es utilizada en la comunidad profesional que los produce para ilustrar el uso y aplicación de las leyes, establecer precedentes y registrar casos anteriores que se utilizan como jurisprudencia en el tratamiento de hechos similares (lo cual se advierte desde el título mismo de la publicación, LexisNexis: Jurisprudencia Argentina). Todo ello en el marco formal definido por lo que el autor presenta como mundo modelo de derechos y obligaciones, permisos y prohibiciones.

En este sentido, y siguiendo el modelo de contextualización presentado más arriba, los casos legales se asocian tanto con la negociación de justicia como con la educación legal.

Los propósitos comunicativos de los casos legales son, pues, constituir auténticos registros de juicios anteriores (acervo de hechos, razonamientos, sentencias, etc.); servir como precedentes para casos futuros (recopilación de evidencias favorables y/o contrarias a un argumento y/o decisión); actuar como recordatorios para los expertos legales, e ilustrar ciertos puntos de la ley (la ley también se aprende desde su aplicación).

En lo atinente a la interpretación estructural de los casos legales, los mismos presentan una estructura cognitiva típica expresada en una serie de actos o movimientos más o menos obligatorios que dependen del propósito comunicativo seleccionado. Dichos actos pueden sintetizarse en: 1) identificación del caso (rótulo/nombre; por ejemplo, Sánchez contra Martínez sobre daños y perjuicios); 2) establecimiento de los hechos del 


\section{GRÁFICO 2}

Contextualización de los casos legales

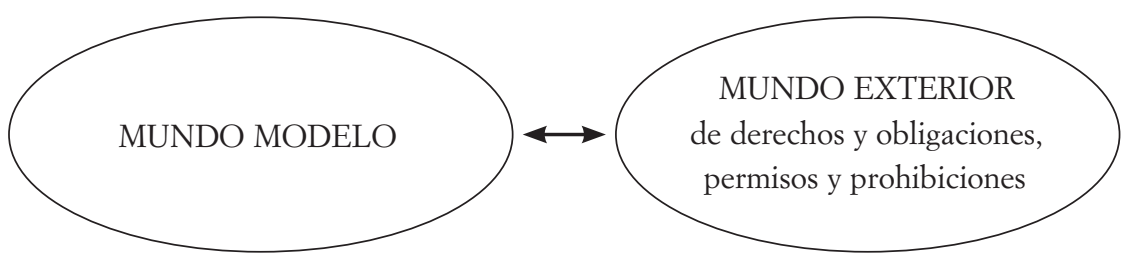

CASOS LEGALES

Procesos de argumentación legal destinados a la negociación de justicia y a la educación legal.

Suponen ejemplos de interacción concreta entre el mundo exterior y el mundo modelo

caso; 3 ) argumentación del caso (expresión de la historia del caso/presentación de argumentos/establecimiento de ratio decidendi, asociado al propósito de servir como precedente para casos futuros), y 4) pronunciamiento de sentencia (se trata de textos jurídicos fuertemente argumentativos que despliegan variados recursos y estrategias a favor de lo sentenciado). Esta subjetividad legal es precisamente la que humaniza los textos en detrimento de la pretendida objetividad que los atraviesa ${ }^{17}$.

En relación a la forma en que se construyen los argumentos que sirven de fundamentación a los casos legales, Perelman sostiene que los acuerdos se organizan a partir de «hechos, verdades y presunciones; por otro lado, de valores, jerarquías y lugares de preferencia» ${ }^{18}$.

\section{EL TEXTO JUDICIAL COMO TEXTO DE PODER}

En este punto sólo deseamos señalar algunas ideas que se desprenden del análisis efectuado más arriba, que al identificar los elementos estructurales del discurso jurídico en el marco de situaciones comunicativas particulares posibilita la percepción de los textos judiciales como textos de

${ }_{17}$ M. Pardo, Derecho y lingüística. Cómo se juzga con palabras. Análisis lingüístico de sentencias judiciales, Buenos Aires, 1992, p. 148.

${ }^{18}$ R. Marafioti, op. cit., p. 103. 
poder. Nuestro objetivo al respecto se limitará a señalar algunos aspectos para futuros análisis.

Cabe aclarar que no desconocemos que las implicaciones de colocar a los textos en una comunidad de pertenencia específica —atendiendo a todos sus condicionantes desde la mirada de los actores- ha sido abordada mayormente por la perspectiva socio-cognitiva, la cual no ha sido desarrollada en este trabajo de carácter introductorio. No obstante lo dicho, creemos importante poner de manifiesto que el texto judicial es un texto de poder, con implicancias ideológicas aplicables a un contexto determinado y tendente a modelar las experiencias.

Según la propuesta de Pardo ${ }^{19}$, que retoma a Van Dijk y a Fairclough, el poder se define por el acceso al control de la información, de los recursos y de las acciones del grupo dominante sobre el dominado. En términos de Foucault, la concepción tradicional del poder remite al «poder como mecanismo esencialmente jurídico, lo que dice la ley, lo que prohíbe, lo que dice no» ${ }^{20}$. El mismo autor explica que esa concepción del poder le sirvió cuando escribió La bistoria de la locura en la época clásica ${ }^{21}$, pero no es la misma que utiliza en Vigilar y castigar. Nacimiento de la prisión ${ }^{22}$, donde sustituye el esquema jurídico y negativo por otro técnico y estratégico.

Es significativa, en esta instancia, la consideración del contexto donde se aplica o se pone en funcionamiento el lenguaje jurídico. En este sentido, los ámbitos de poder estatal como el ejecutivo, legislativo y judicial recurren a normas que estructuran el comportamiento de quienes interactúan en esas áreas; estableciendo desde esta perspectiva roles inherentes a unos y otros que hacen a la rigidez y a la jerarquización de los comportamientos.

\section{CONSIDERACIONES FINALES}

Tal como se enuncia al comienzo de la presentación, nos propusimos aproximarnos al lenguaje jurídico desde una mirada que fuera más allá de las funciones y estructura del lenguaje y que pudiera explicitar la importancia de la dimensión contextual, de ahí el enfoque sistémico aplicado al estudio de un lenguaje complejo.

${ }_{19}$ Ibid., p. 53.

${ }^{20}$ M. Foucault, Microfísica del poder, Madrid, 2004, p. 154.

${ }^{21}$ Bogotá, 1998.

${ }^{22}$ Madrid, 1996. 
El aporte significativo de los artículos leídos y autores escogidos nos permitieron hacer un mapeo del texto jurídico a través del enfoque situacional en dos géneros que, básicamente, nos sirvieron para poder constatar la aplicabilidad de las conceptualizaciones al momento del análisis del lenguaje legal.

La operatividad teórica de las nociones de género y registro resultaron funcionales a la intención con que pretendimos analizar lo que ha sido definido como un lenguaje de especialidad, fundamentalmente interesada en la identificación de los dos componentes del contexto que reverberan e impactan sobre el texto.

En lo que aparece como el nudo del trabajo, utilizamos dos géneros específicos como son los casos y las disposiciones legales, transformando el texto en un laboratorio donde pudimos explorar su configuración contextual y su estructura cognitiva, sin dejar de lado la inquietud en la pretensión comunicativa.

La última parte de la presentación fue pensada como una invitación a futuras incursiones en el texto jurídico. Su asociación con el concepto de poder abre un abanico de posibilidades y perspectivas analíticas que, si bien exceden nuestro abordaje, no quisimos dejar de mencionar a fin de completar esta serie de herramientas analíticas del lenguaje jurídico en su especificidad.

\section{BIBLIOGRAFÍA}

Bhatia, V., «Legal discourse in professional settings», en V. BHAтtA (ed.), Analysing Genre: language use in professional settings, 1998, pp. 101-144.

Carrió, G., Notas sobre derecho y lenguaje, Buenos Aires, 1979.

Duarte, C., y Martínez, A., El lenguaje jurídico, Buenos Aires, 1995.

Dworkin, R., Los derechos en serio, Buenos Aires, 1993.

Eggins, S., y Martin, J., «Géneros y registros del discurso», en T. van DijK (comp.), El discurso como estructura y proceso. Estudios del discurso I. Una introducción multidisciplinaria, vol. 1, Barcelona, 2003, pp. 335-372.

FerReres Comella, V., El principio de taxatividad en materia penal y el valor normativo de la jurisprudencia (una perspectiva constitucional), Madrid, 2002.

Fóscolo, N., y Schilardi, M., Materialidad y poder del discurso. Decir y hacer jurídicos, Mendoza, 1996.

Foucault, M., La historia de la locura en la época clásica, Bogotá, 1998.

- Microfísica del poder, Madrid, 2004.

- Vigilar y castigar. Nacimiento de la prisión, Madrid, 1996. 
LeXIs NeXIs, Jurisprudencia Argentina, Compraventa, 15 de enero de 2003, JA 2003-1, fascículo 3.

Linares Quintana, S., Tratado de interpretación constitucional, Buenos Aires, 1998.

Marafioti, R., Los patrones de la argumentación. La argumentación en los clásicos y en el siglo XX, Buenos Aires, 2003.

Pardo, M., Derecho y lingüística. Cómo se juzga con palabras. Análisis lingüístico de sentencias judiciales, Buenos Aires, 1992.

PÉrez, S., «Voces en el Parlamento», en A. RAITER et al., Discurso y ciencia social, Buenos Aires, pp. 135-142.

Pérez, S., y Zullo, J., «Subjetividad, discurso y género: una propuesta metodológica», Mora. Revista del Instituto Interdisciplinario de Estudios de la Mujer, núm. 5 (1999), pp. 113-121.

Rosetti, M., y RosetTi, R., Pautas pragmáticas y sintácticas para redactar una ley, Buenos Aires, 1997.

SANTIBÁÑEZ YÁÑEZ, C., «La argumentación jurídica como razonamiento situado», Discurso, núm. 2 (2001), pp. 33-47.

Swales, J., Genre Analisis. English in academia and research settings, Cambridge, 1998.

VON Wright, G., Norma y acción. Una investigación lógica, Madrid, 1970. 